\title{
HINS Superconducting Lens and Cryostat Performance
}

\author{
T. M. Page, J. DiMarco, Y. Huang, D. F. Orris, M. A. Tartaglia, I. Terechkine, J. C. Tompkins
}

\begin{abstract}
Fermi National Accelerator Laboratory is involved in the development of a $60 \mathrm{MeV}$ superconducting linac. This linac is part of the High Intensity Neutrino Source (HINS) R\&D Program. The initial beam acceleration in the front end section of the linac is achieved using room temperature spoke cavities, each of which is combined with a superconducting focusing solenoid. These solenoid magnets are cooled with liquid helium at $4.5 \mathrm{~K}$, operate at $250 \mathrm{~A}$ and have a maximum magnetic field strength of 7.5 T. A prototype solenoid cryostat was built and tested at the Fermilab Magnet Test Facility. This paper discusses the test results of the prototype and compares the measured and estimated performance of the cryostat. We also present the methods and results for measuring and fiducializing the axis of the solenoid lens.
\end{abstract}

Index Terms-Alignment, Cool-down, Cryostat, HINS, Magnet, Superconducting Solenoid.

\section{INTRODUCTION}

$\mathrm{F}$ ERMILAB is designing and building the front end of a high - power $\mathrm{H}^{-} \mathrm{RF}$ linac as part of the High Intensity Neutrino Source (HINS) R\&D effort [1]. Initial acceleration of the beam is achieved by a Radio Frequency Quadrupole (RFQ) from 0.05 MeV to 2.5 MeV and room temperature (RT) crossbar H-type (CH) accelerating cavities up to $10 \mathrm{MeV}$ [2], [3]. Each of these cavities is coupled with a superconducting solenoid magnet to provide beam focusing [4], [5]. A layout of the linac is shown in Fig. 1. This paper presents results for the RT-section (a.k.a., CH-section) prototype cryostat and focusing lens, designated HCH-P-001; subsequent SSR1 and SSR2 sections will utilize stronger focusing solenoids assembled with superconducting spoke resonator cavities into long cryostats [6].

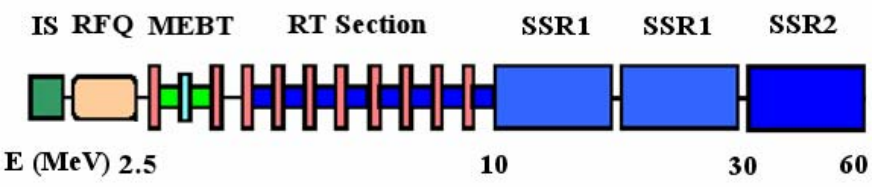

Fig. 1. HINS front end layout schematic.

Manuscript received 19 August 2008. Work supported by the U.S. Department of Energy under contract No. DE-AC02-07CH11359.

T. M. Page, J. Dimarco, Y. Huang, D. F. Orris, M. A. Tartaglia, I. Terechkine and J. C. Tompkins are with Fermi National Accelerator Laboratory, P.O. Box 500, Batavia, IL 60510 USA. Corresponding author T. M. Page; phone: 630-840-8019; e-mail: tpage@fnal.gov.
There are 19 individually cryostated solenoid magnets installed up through the RT-section of the linac. Two versions of solenoid magnets are required, one with integrated horizontal and vertical dipole steering-correction coils and one without. The solenoids without dipoles are designated as Type-1 magnets and the solenoids with steering-correction coils are designated as Type- 2 magnets. The cryostats for each type are similar with the exception of the power leads. Due to the addition of the dipole correctors, Type-2 cryostats require two extra pairs of power leads for a total of six leads. The prototype cryostat contains magnet HINS_CH_SOL_01, which was assembled into a helium vessel and cold tested in a vertical dewar prior to installation in the cryostat [7]. This magnet was the first prototype Type- $1 \mathrm{CH}$-section solenoid built as part of the HINS magnet R\&D program [8].

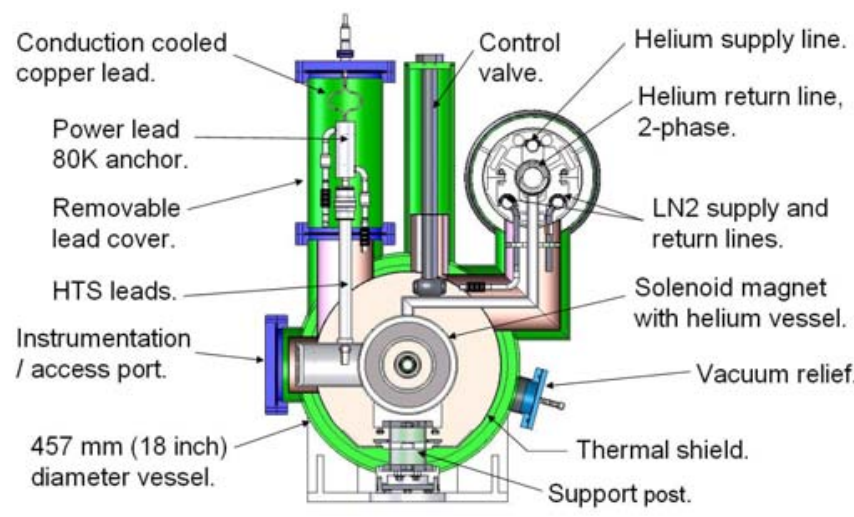

Fig. 2. Cross section through a Type-1 solenoid cryostat.

A cross section of the production Type- 1 solenoid cryostat is shown in Fig. 2 [9]. The prototype cryostat differs from the production Type- 1 cryostat in two ways. First, the HTS leads which will be used in production were not available for integration into the prototype and therefore standard vapor cooled leads were installed in their place. Second, the liquid nitrogen control valve (needed for HTS leads) was left out of the prototype. This empty tube was then used to route all of the instrumentation out of the cryostat.

\section{CRYOSTAT ASSEMBLY AND INSTRUMENTATION}

\section{A. Magnet Installation}

Components and sub-assemblies for the cryostat were fabricated by both outside vendors and Fermilab shops with the final assembly of the cryostat being completed at Fermilab. Total elapsed assembly time was approximately 8 weeks with one technician performing the assembly work and 
coordinating additional services like welding, brazing and leak checking. Fig. 3 shows the magnet assembly installed in the vacuum vessel before MLI and thermal shield installation.

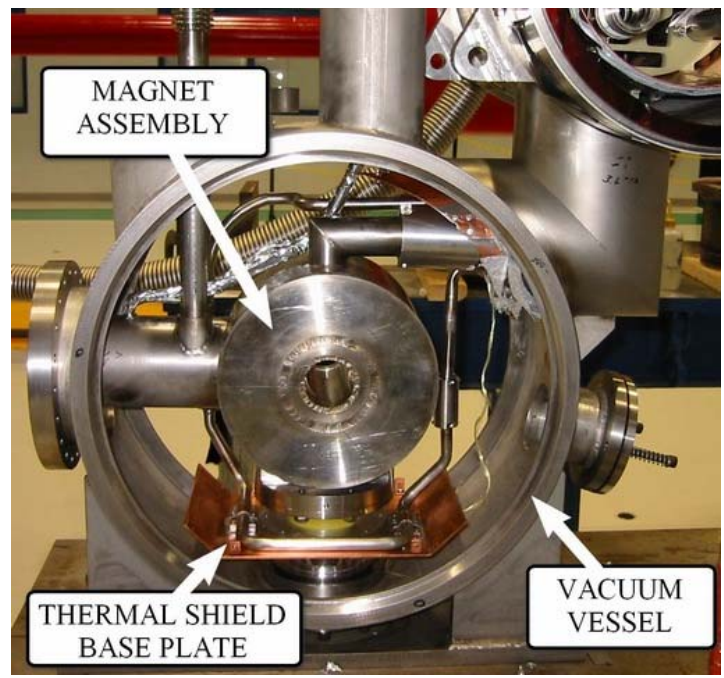

Fig. 3. Solenoid magnet assembly installed in vacuum vessel.

Positioning of the magnet assembly in the vacuum vessel at this stage is purely mechanical. Magnetic measurements and optical survey are not performed until after the cryostat is completely closed. The beam center height is set by the support post and is nominally centered to the inside diameter of the vacuum vessel by design. Rotation is set by squaring up the end plates of the magnets with the end ring of the vacuum vessel. Once set, a mounting ring and set screws are used to lock the magnet into position. Adjustment of the magnet after installation in the linac is performed by adjusting the entire cryostat using specially designed support girders with 6 degree of freedom adjustment.

\section{B. Instrumentation and Test Stand}

The cryostat assembly was instrumented with six Lakeshore thermometers: four Platinum RTD's on the thermal shield and two Cernox sensors in the helium space. The shield RTD's were placed at the following locations to determine the temperature distribution around the shield: one on the base plate, one on the body, one on one of the end walls and one on the cryogenic distribution header. The shield base plate shown in Fig. 3 serves as the main thermal anchor for the shield, the mechanical support for the shield and the thermal intercept for the support post. Threaded fasteners and tack welds attach the shield body and end walls to the base plate. The cryogenic distribution header contains a shield around the piping which is not attached to the main cryostat shield. Instead, two copper thermal straps were soldered between the liquid nitrogen return line and the cryogenic header thermal shield.

This cryostat is very different from previous magnet cryostats built and tested at Fermilab. A new stand for testing these 19 magnets was not within the budget and time scale for the HINS effort so an adapter was designed and built in order to utilize one of the existing (Tevatron magnet) test stands at the Fermilab Magnet Test Facility (MTF). The feed box provides insulating vacuum and supplies liquid nitrogen and single phase helium. Turn-around pipes cap the end of the distribution header, and return two-phase helium through a JT valve in the adapter. Existing feed box instrumentation measures $\mathrm{He}$ and N2 temperatures and pressures at the adapter interface. Fig. 4 shows the completed cryostat assembly and adapter mounted to the test stand. Note that space is allocated for close, stable mounting of alignment stages on both sides of the cryostat (see section IV).

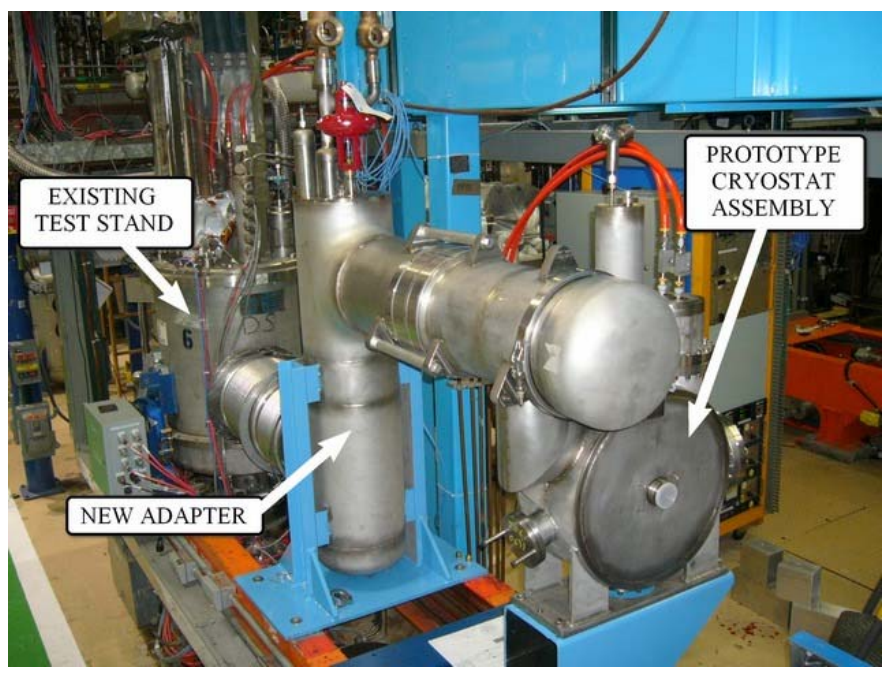

Fig. 4. Cryostat and adapter mounted to test stand.

\section{CRYostat PeRformanCE}

One test cycle of the prototype cryostat has been completed. Testing began with interconnections and system leak checks in June 2007. Warm alignment and survey were followed by a cool down, cold alignment and survey, power and quench performance tests and warm-up to room temperature. This test cycle will be repeated in order to measure the reproducibility of the solenoid alignment.

\section{A. Magnet Cool-down}

Cool-down of the magnet was achieved by first cooling the thermal shield and then the magnet. Helium boil-off gas is all taken through the vapor cooled power leads; in the production cryostat with HTS leads, a small cool-down/warm-up line will be introduced. The magnet cool-down rate was approximately 60 to $70 \mathrm{~K}$ per hour at a flow rate of $0.2 \mathrm{~g} / \mathrm{sec}$ of helium.

\section{B. Thermal Shield}

The thermal shield was cooled down with a flow of 0.91 $\mathrm{g} / \mathrm{sec}$ (100 SCFH). Once cold, the minimum LN2 flow necessary to maintain steady shield temperatures was determined to be $0.55 \mathrm{~g} / \mathrm{sec}$ (60 SCFH). The temperature distribution across the thermal shield is shown in Table I.

These results show that thermal connections between shield parts were very good except for the distribution piping. When the piping shield was mounted there was a large mismatch at the junction of the piping shield and the body shield. This made it impossible to connect the two shields, so the piping shield is only cooled through two copper braids soldered between the LN2 return pipe and the shield. Although a $15 \mathrm{~K}$ temperature difference is acceptable in this region, the piping 
thermal shield connection will be improved for the production magnets in an effort to reduce this temperature difference below $10 \mathrm{~K}$.

TABLE I Thermal Shield Temperature Distribution

\begin{tabular}{lcc}
\hline \hline RTD location & $\begin{array}{c}\text { Temperature after } \\
\text { initial cool-down } \\
\text { (Magnet at 300K) }\end{array}$ & $\begin{array}{c}\text { Temperature } \\
\text { after cool-down } \\
\text { of magnet }\end{array}$ \\
\hline Feed Box Supply & $91 \mathrm{~K}$ & $91 \mathrm{~K}$ \\
\hline Base plate & $93 \mathrm{~K}$ & $93 \mathrm{~K}$ \\
Body & $94 \mathrm{~K}$ & $93 \mathrm{~K}$ \\
End wall & $95 \mathrm{~K}$ & $94 \mathrm{~K}$ \\
Distribution pipes & $124 \mathrm{~K}$ & $108 \mathrm{~K}$ \\
\hline \hline
\end{tabular}

The warm bore tube through the magnet does not have a thermal shield. Instead, 40 layers of MLI were wrapped around this tube. During operation the temperature within the warm bore tube was measured to be approximately $1 \mathrm{~K}$ lower than other locations on the surface of the vacuum vessel.

\section{Power Leads}

Helium gas flow through each power lead was controlled and monitored manually using a rotameter. There are three operating conditions of the leads that are of interest, (1) standby with no current, (2) steady operating current and (3) current ramp-up. In standby condition, flows were adjusted down to $0.014 \mathrm{~g} / \mathrm{sec}$ per lead to prevent frost and minimize helium consumption. This is not as critical for test stand operation as it is for linac operation where there will be a total of 24 pairs of vapor cooled leads installed. During testing of the production magnets this standby minimum flow condition will be investigated further.

Under steady conditions operating at a current of $235 \mathrm{~A}$, the leads were stable at a minimum flow of $0.027 \mathrm{~g} / \mathrm{sec}$ per lead. Voltage across leads at these conditions was $65 \mathrm{mV}$. The lead flow was adjusted to as low as $0.020 \mathrm{~g} / \mathrm{sec}$ per lead but lead voltages were slowly rising and this flow was determined unacceptable.

A flow of $0.044 \mathrm{~g} / \mathrm{sec}$ per lead was required during magnet ramping to prevent quenching at low current. Various ramp rates were investigated from $1 \mathrm{~A} / \mathrm{sec}$ to $8 \mathrm{~A} / \mathrm{sec}$ with the same results. The lead flow could be reduced to the steady operating condition of $0.027 \mathrm{~g} / \mathrm{sec}$ per lead once the operating current was reached.

\section{Quench Performance}

After the prototype solenoid was welded into its helium vessel, it reached a quench current of $260 \mathrm{~A}$ after two retraining quenches when tested in a vertical dewar at $4.21 \mathrm{~K}$ [7]. Helium was at $4.58 \mathrm{~K}$ in the cryostat test, due to the distribution at 22 psia pressure, which is higher than is expected for the actual linac. The expected quench current due to this change in temperature is $247 \mathrm{~A}$. At a low ramp rate of $1 \mathrm{~A} / \mathrm{s}$, the cryostated solenoid quenched reproducibly at 243 A, which is in good agreement with the prediction. Ramp rate dependence was weak: the quench current dropped to $238 \mathrm{~A}$ at a current ramp rate of $8 \mathrm{~A} / \mathrm{s}$. The temperature of the helium in the cryostat following a quench rose to approximately $7 \mathrm{~K}$ to 8 $\mathrm{K}$. The helium temperature recovered in approximately 1 minute.

As discussed above, when lead flows were too low the solenoid quenched repeatedly at low current: at about $85 \mathrm{~A}$ for lead flows of $0.014 \mathrm{~g} / \mathrm{s}$, and at 216 A with flows of twice this value.

\section{Alignment}

Alignment of the HINS solenoids is currently being done with the Single Stretched Wire (SSW) system [10], which is used at Fermilab for most magnet alignment tasks. A 100 micrometer $\mathrm{CuBe}$ wire is stretched through the length of the magnet, connected to precision (1 micrometer accuracy) motion stages at both ends. The return wire of the flux loop lies stationary on the bottom of the beam pipe. Using co- and counter- directional stage motions (i.e. stages moving in the same or opposite directions), the $x, y$ coordinates of the magnet center and the pitch, yaw of the magnet axes can be determined; the stages are then moved so that the wire endpoints are coincident with the magnetic axis and then surveyed to external fiducials. The SSW setup is shown in Fig. 5.

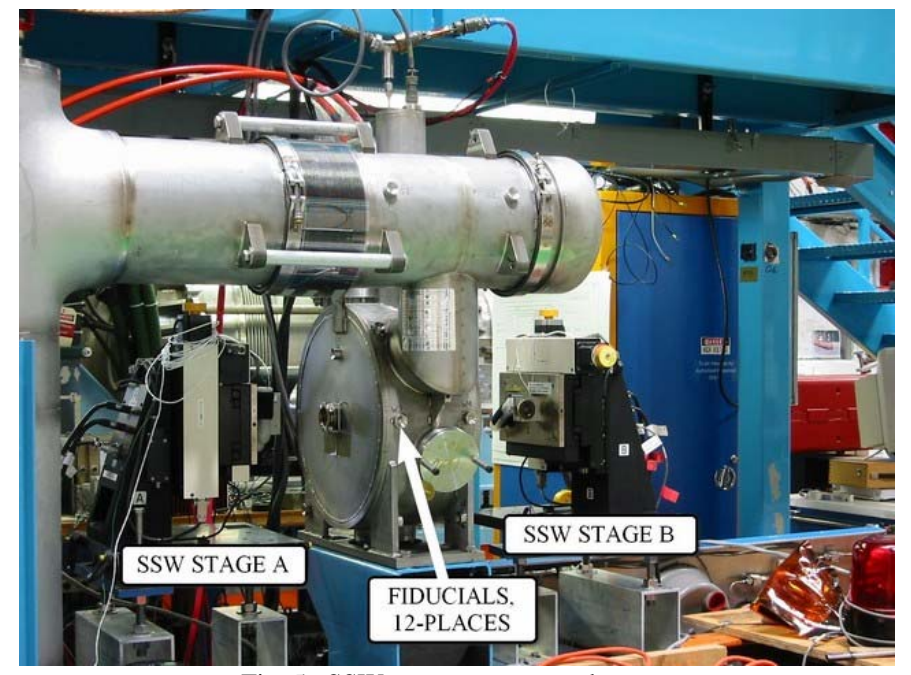

Fig. 5. SSW setup on test stand.

For the solenoids, alignment consists of balancing flux changes for various stage motions. For example, to determine the solenoid pitch angle, the wire would be displaced to various pitch angles (i.e. the stages would move counterdirectionally in the +/- vertical direction) and co-directional horizontal measurements would be performed. The stage pitch angle which produces no flux change for horizontal motions coincides with the solenoid pitch. Likewise, to determine yoffset, the stages are moved to various y-offsets (i.e. codirectional, vertical stage motion) and counter-directional horizontal measurements performed. The y-position at which no flux change is measured for the counter-directional, horizontal stage motions coincides with the vertical solenoid center. In practice, a fit is made of the results at more than one displacement and the axis position is determined from interpolation. Determination of yaw and $\mathrm{x}$-offset follows similarly. 
The reproducibility in the alignment measurements is better than 15 micrometers cold and about 50 micrometers warm. The measurements are particularly challenging with this technique given the small $(20 \mathrm{~mm})$ warm bore aperture.

HCH-P-001 had its alignment measured at room temperature, both with and without vacuum, as well as at cryogenic temperature. Room temperature measurements are made with $0.5 \mathrm{~A}$ AC current, and the cold measurements are at 50-150 A DC. The change of solenoid axis position during cool-down is shown in Fig. 6.

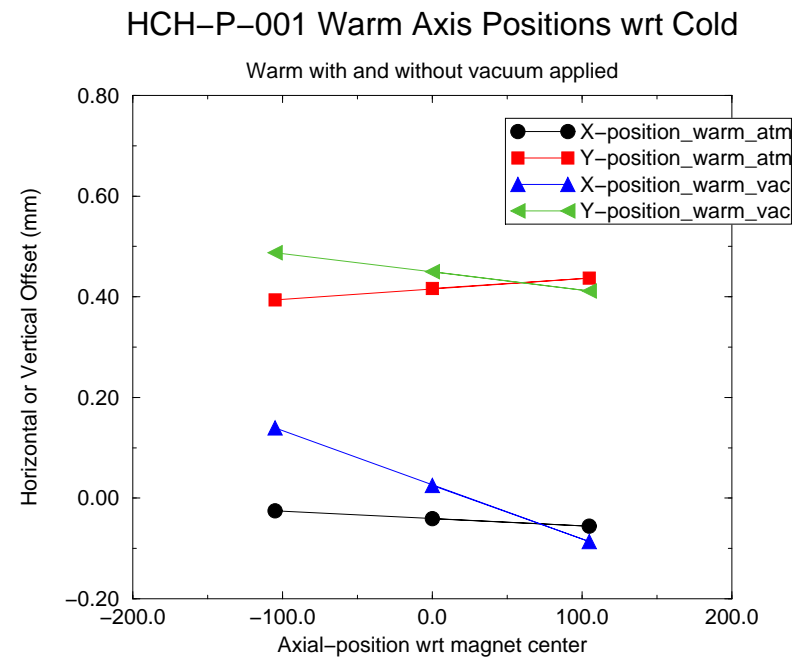

Fig. 6. Position of solenoid axes warm, with and without vacuum, with respect to the cold axes measured at $4.5 \mathrm{~K}$ and zero current.

There is a vertical shift downward of about $0.45 \mathrm{~mm}$ during cool-down, and close to zero change horizontally. This compares well with simulations that showed a $0.53 \mathrm{~mm}$ downward shift after cool-down. There are also small changes in yaw and pitch with application of vacuum, but these begin to border on measurement uncertainties.

Changes in axes positions from powering the magnet to various currents cold are shown in Fig. 7. Motions of about 50 micrometers in both horizontal and vertical directions are observed over the change from 50 to $150 \mathrm{~A}$.

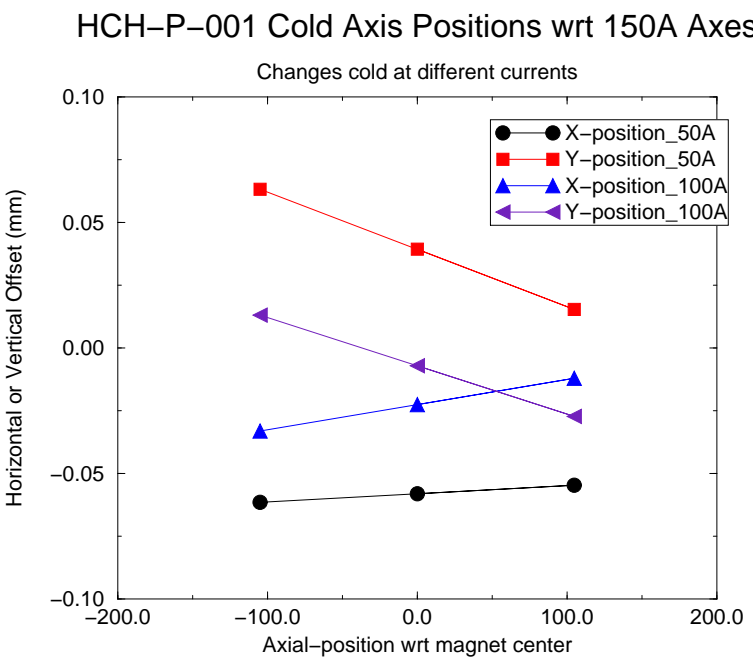

Fig. 7. Change in solenoid axis position from vacuum and powering.

\section{CONCLUSION}

A single test cycle of the $\mathrm{CH}$-section prototype cryostat has been performed in the Fermilab Magnet Test Facility. The cryostat worked well in this test with some minor improvements being identified for incorporation into the production cryostats. A first look at alignment indicates that lens magnetic axis measurements can be made at the required level of precision both warm and cold; they indicate the cooldown shrinkage to be about as expected, and suggest the changes of alignment with current are small and acceptable. Additional thermal test cycles will be conducted in the near future to determine the reproducibility of the warm and cold lens alignment. Assembly of Type- 1 and Type- 2 production $\mathrm{CH}$-section cryostated lenses is expected to begin this fall.

\section{ACKNOWLEDGMENT}

The authors wish to thank S. Sanchez for his technical expertise in assembling the prototype cryostat, C. Reid for development of the adapter design, W. Gatfield and D. Watkins for extensive and artful welding, C. Hess for his expert help with adapter assembly and leak checking, F. Lewis for providing broad electrical support, S. Kotelnikov and W. Shappert for data acquisition development, the Fermilab alignment crews for prompt service and precise results, and the MTF cryogenics team for expert help with many vacuum and cryogenic operations issues.

\section{REFERENCES}

[1] P. N. Ostroumov, K. W. Shepard, G. W. Foster, I. V. Gonin, and G. V. Romanov, "Front end design of a multi-GeV H-minus linac," in Proceedings of PAC-05, May 2005, pp. 3286-3288.

[2] P. N. Ostroumov, et al, "Application of a New Procedure for Design of 325 MHz RFQ,” Journal of Instrumentation 1, P04002, 2006, Available: http://www.iop.org/EJ/abstract/1748-0221/1/04/P04002/.

[3] L. Ristori, et al, "Fabrication and Test of the First Normal-Conducting Crossbar H-Type Accelerating Cavity at Fermilab for HINS," in Proceedings of PAC-07, June 2007, pp. 2292-2294.

[4] G. Davis, V.V. Kashikhin, T. Page, I. Terechkine, J. Tompkins, and T. Wokas, "Designing Focusing Solenoids for Superconducting RF Accelerators," IEEE Transactions on Applied Superconductivity, vol. 17, no. 2, June 2007, pp. 1221-1224.

[5] I. Terechkine, V.V. Kashikhin, T. Page, M. Tartaglia, J. Tompkins, "Focusing Solenoid for the Front End of a Linear RF Accelerator," in Proceedings of PAC-07, June 2007, pp. 473-475.

[6] G. Apollinari, et al., "Hins Linac Front End Focusing System R\&D," IEEE Transactions on Applied Superconductivity, vol. 19, submitted for publication.

[7] C.Hess, et al., "Focusing Solenoid HINS_CH_SOL_01 Fabrication Notes and Test Results,” Fermilab technical note TD-07-006, Fermilab, May 2007, Available: http://tdserver1.fnal.gov/tdlibry/TDNotes/2007\%20Tech\%20Notes/TD-07-006.pdf

[8] M.A.Tartaglia, et al., "Test Results for HINS Focusing Solenoids at Fermilab,” IEEE Transactions on Applied Superconductivity, vol. 19, submitted for publication.

[9] T. M. Page, et al., "High Intensity Neutrino Source Superconducting Solenoid Cryostat Design,” in Advances in Cryogenic Engineering 53A, edited by J. G. Weisend II, American Institute of Physics, New York, 2008, pp. 341-348.

[10] J. DiMarco et al., "Field Alignment of Quadrupole Magnets for the LHC Interaction Regions," IEEE Transactions on Applied Superconductivity, vol. 10, no. 1, March 2000, pp. 127-130. 